\title{
Neurocysticercosis: diagnostic dilemma
}

\author{
Joyce HM Cheng *, MB, ChB, Eric MW Man, MB, ChB, FRCR, SY Luk, MB, BS, FRCR, Wendy WC Wong, MB, BS, FRCR
}

\author{
Department of Radiology, Pamela Youde Nethersole Eastern Hospital, Chai Wan, Hong Kong
}

*Corresponding author: chm915@ha.org.hk

Hong Kong Med J 2016;22:616-8

DOI: $10.12809 / \mathrm{hkmj} 154524$

\section{Case report}

A 7-year-old Nepalese boy with unremarkable past health was admitted in July 2013 with a 2 -week history of headache, dizziness, and vomiting that subsided spontaneously. On admission, he was afebrile with unremarkable neurological and fundal examination.

He had been living in Nepal until the age of 5 years and then immigrated to Hong Kong. He recently travelled to Nepal but had otherwise no contact history with pigs or febrile persons.

Blood tests were unremarkable except mildly elevated erythrocyte sedimentation rate of $26 \mathrm{~mm} / \mathrm{h}$. Lumbar puncture yielded normal cerebrospinal fluid (CSF) white cell count, and protein and glucose level. Culture of blood and CSF was negative.

Initial contrast computed tomographic brain showed two subcentimetre $(7 \mathrm{~mm}$ and $9 \mathrm{~mm}$ ) left parieto-occipital adjacent rim-enhancing lesions (Fig a) with T1-weighted hypointense and T2weighted/fluid-attenuated inversion recovery hyperintense signal on contrast magnetic resonance imaging (MRI) [Fig b]. Significant perifocal oedema and mass effect with compression on the left occipital horn was noted. Magnetic resonance spectroscopy showed a significant lipid-lactate peak (Fig c), decreased N-acetylaspartate, and no elevated choline. No amino acid peak was identified. Perfusion imaging with arterial spine labelling showed no elevated regional cerebral blood flow (Fig d). Contrast MRI of the spine showed no leptomeningeal enhancement, intradural nor extradural spinal mass lesions.

Subsequent extensive investigations of blood (including anti-Taenia solium immunoglobulin G), CSF, urine and stool for tuberculosis and T solium were performed, and all results were negative.

Overall review of the clinical presentation, travel history, laboratory and imaging findings, and the two cerebral rim-enhancing lesions were suggestive of an infective process, in particular, neurocysticercosis or tuberculosis.

After thorough investigations and interdepartmental discussions, it was decided to treat the patient as a probable case of neurocysticercosis and a course of empirical albendazole and prednisolone was prescribed.

The patient remained asymptomatic after treatment. Follow-up MRI performed after 2 and 5 months (Fig e) showed reduction in size of the two cerebral rim-enhancing lesions ( $3 \mathrm{~mm}$ and $1 \mathrm{~mm}$ ), as well as the extent of perifocal oedema.

\section{Discussion}

Neurocysticercosis is a parasitic infection of the central nervous system by $T$ solium (ie pork tapeworm) usually through accidental ingestion of contaminated food containing its eggs. It has been a diagnostic challenge in developed areas owing to its infrequent occurrence, low sensitivity of serological tests, and highly variable and non-specific manifestations, both clinically and radiologically. It is further complicated by clinical and radiological features that overlap considerably with tuberculosis infection, particularly in tuberculosis-endemic areas.

A few international diagnostic criteria for neurocysticercosis have been proposed, and aim to stratify the diagnostic strength based on the interpretation of clinical, radiological, serological, and epidemiological information. One of the most widely accepted revised criteria was proposed by Del Brutto et al in 2001, ${ }^{1}$ and classifies patients into two degrees of diagnostic certainty-definitive or probable. Definitive diagnosis requires the presence of one absolute criterion or two major plus one minor epidemiological criteria. Probable diagnosis can be made if there are one major plus two minor criteria; one major plus one minor and one epidemiological criteria; or presence of three minor plus one epidemiological criteria. Four categories of criteria based on their diagnostic strength are shown in Table a. ${ }^{1}$

The diagnostic dilemma in our index case was the lack of characteristic imaging features and positive serological proof, hence difficult differentiation from tuberculosis infection, i.e. tuberculomas. Other common differential diagnoses of cystic lesions include pyogenic abscesses and metastases. Both were unlikely in our patient given the aseptic clinical presentation, normal blood and CSF profiles, absence of a known primary and lack of associated radiological findings (eg cerebritis, ventriculitis or meningeal enhancement). Biopsy or excision of the cerebral cystic lesions might offer a straightforward histopathological diagnosis, however, it is an invasive investigation and imposes potential risks. 

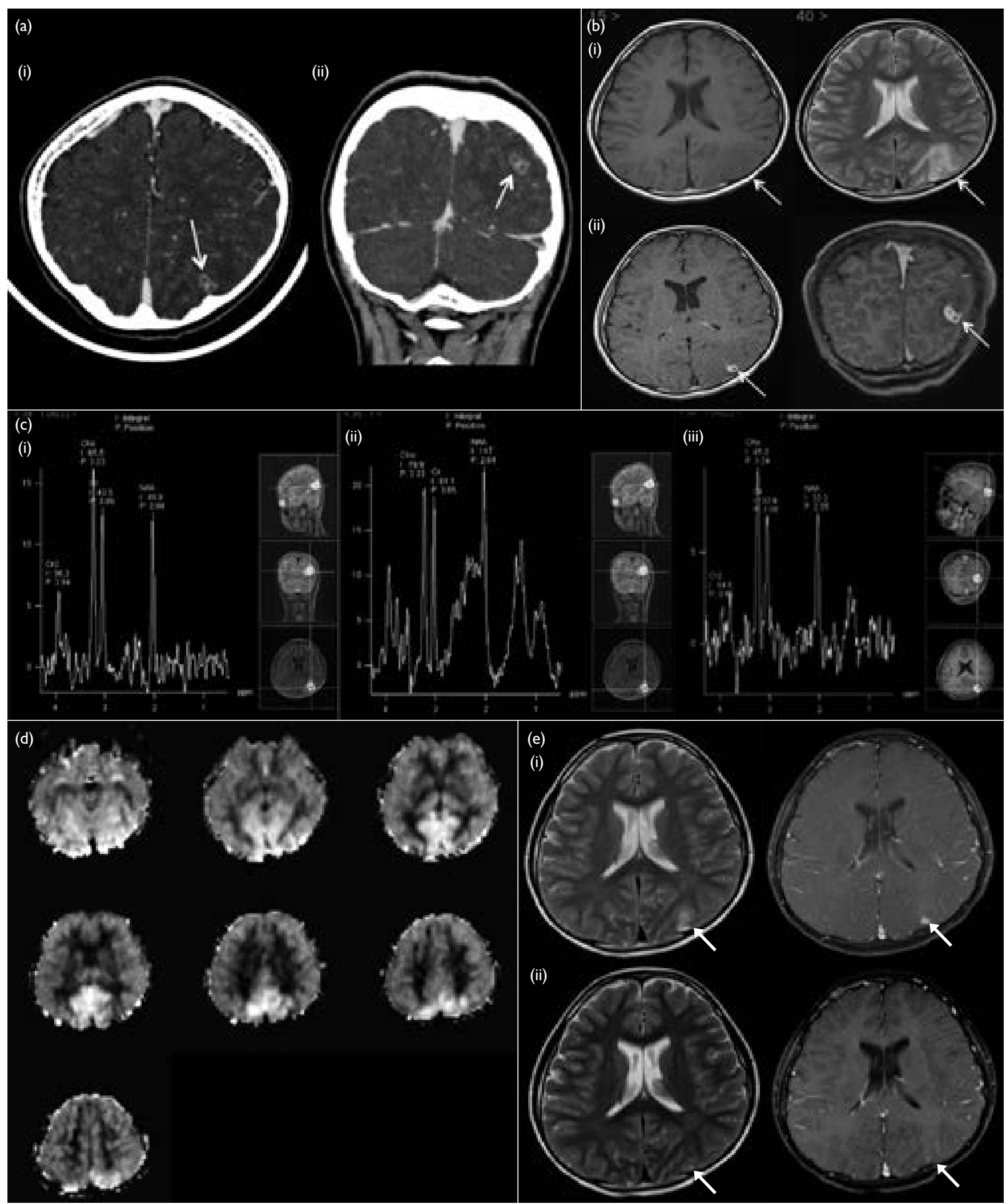

FIG. (a) (i) Axial and (ii) coronal reformatted contrast computed tomography showing two rim-enhancing lesions over left parieto-occipital region (arrows). (b) (i) Magnetic resonance (MR) axial images showing TI-weighted (TIW) hypointense and T2-weighted (T2W) hyperintense lesions over left parieto-occipital region with perifocal oedema (arrows); (ii) post-gadolinium TIW MR axial and coronal images showing rim enhancement of the lesions (arrows). (c) Single-voxel MR spectroscopy of left parieto-occipital lobe lesion of (i) short (TE 30), (ii) intermediate (TE I35), and (iii) long (TE 270) echo showing significantly elevated lipid-lactate (I.3 ppm), with a peak doublet centred at I.3 ppm in TE 30 and TE 270, and inverts in TE I35; N-acetylaspartate peak $(2.0 \mathrm{ppm})$ is decreased; and choline (3.2 ppm) is not elevated. (d) Perfusion imaging in arterial spine labelling showing no significantly elevated regional cerebral blood flow over the left parieto-occipital lesions. (e) T2W and post-gadolinium TIW MR axial images at (i) 2-month and (ii) 5-month intervals showing gradual reduction in the size of the two rim-enhancing lesions over parieto-occipital region (arrows) 
TABLE. (a) Four categories of criteria based on their diagnostic strength'

\begin{tabular}{ll}
\hline Category & Diagnostic strength \\
\hline Absolute & $\begin{array}{l}\text { Histological confirmation of parasite from brain/spinal cord lesion biopsy, radiological demonstration of cystic lesions with } \\
\text { scolex, and fundoscopic direct visualisation of subretinal parasites }\end{array}$ \\
\hline Major & $\begin{array}{l}\text { Highly suggestive imaging features: cyst without scolex, enhancing or calcified lesion, positive serum anti-cysticercus } \\
\text { antibodies, resolution of cystic lesions after therapy (albendazole or praziquantel), and spontaneous resolution of small single } \\
\text { enhancing lesion }\end{array}$ \\
\hline Minor & $\begin{array}{l}\text { Lesions compatible with neurocysticercosis on neuroimaging, clinical manifestations suggestive of neurocysticercosis, } \\
\text { positive CSF enzyme-linked immunosorbent assay, and cysticercosis outside CNS }\end{array}$ \\
\hline Epidemiological & $\begin{array}{l}\text { Evidence of household contact with Taenia solium infection, individuals from or living in endemic areas of cysticercosis, and } \\
\text { history of frequent travel to disease-endemic areas }\end{array}$ \\
\hline
\end{tabular}

Abbreviations: CNS = central nervous system; CSF = cerebrospinal fluid

TABLE. (b) MR imaging features which may help in distinguishing neurocysticercosis from tuberculosis ${ }^{2-5}$

\begin{tabular}{|c|c|c|}
\hline & Neurocysticercosis & Tuberculosis \\
\hline Margin & Regular & Irregular with conglomeration \\
\hline Size & Smaller $(<20 \mathrm{~mm})$ & Larger $(>20 \mathrm{~mm})$ \\
\hline Signal intensity & T1W hypointense, T2W hyperintense & $\begin{array}{l}\text { T1W and T2W hypo- to iso-intense, often with hyperintense rim, rarely onion-peal } \\
\text { appearance (alternating rings of hypo- and hyper-intense signals) }\end{array}$ \\
\hline DWI & Variable & Variable, those with T2W hyperintense centres usually show restricted diffusion \\
\hline Midline shift & No & Yes \\
\hline MR spectroscopy 5 & $\begin{array}{l}\text { - Elevated succinate (related to aerobic } \\
\text { metabolism of scolices), lactate, and } \\
\text { alanine levels } \\
\text { - Low NAA and Cr levels } \\
\text { - Cho/Cr ratio }<1\end{array}$ & $\begin{array}{l}\text { - Elevated lipid (mycobacteria which mainly composed of lipid), lactate, and Cho } \\
\text { levels (associated benign inflammatory reaction) } \\
\text { - Even lower NAA and Cr levels } \\
\text { - Cho/Cr ratio >1 } \\
\text { - Prominent decrease in NAA/Cr, slight decrease in NAA/Cho }\end{array}$ \\
\hline
\end{tabular}

Abbreviations: Cho = choline; $\mathrm{Cr}=$ creatine; $\mathrm{DW}$ = diffusion-weighted imaging; $\mathrm{MR}=$ magnetic resonance; $\mathrm{NAA}=\mathrm{N}$-acetylaspartate; $\mathrm{TI} \mathrm{W}=\mathrm{TI}$-weighted; $\mathrm{T} 2 \mathrm{~W}=\mathrm{T} 2$-weighted

Attempts have been made in the literature to distinguish cysticercosis and tuberculosis neurological infection by combined interpretation of the clinical, radiological, and serological features. ${ }^{2-4}$

Clinically, tuberculomas are more often associated with increased intracranial pressure and focal neurological deficits, whereas patients with neurocysticercosis are usually less symptomatic or present with seizures. $^{2}$

Radiologically, the imaging findings of neurocysticercosis are variable, depending on the stage and location of infestation. A diagnostic dilemma lies in those who present with intracranial cystic lesions without presence of scolex, and that may be present in both neurocysticercosis and tuberculosis. Table b shows some MRI imaging features that may help distinguish the two diagnoses. ${ }^{2-5}$

Based on the neuroimaging features and the revised diagnostic criteria, a probable diagnosis of neurocysticercosis was made: presence of lesions compatible with neurocysticercosis that resolved after treatment with albendazole, and a history of travelling to an endemic area.

Neurocysticercosis is an uncommon parasitic infection of the central nervous system in Hong Kong and requires a high degree of clinical suspicion for diagnosis. Despite the advances in neuroimaging, accurate diagnosis is still sometimes difficult, which is related to the pleomorphic disease nature and significant overlapping features with tuberculosis. A combination of proper interpretation of diagnostic criteria and imaging findings is helpful in making the diagnosis without invasive and potentially harmful investigations.

\section{References}

1. Del Brutto OH, Rajshekhar V, White AC Jr, et al. Proposed diagnostic criteria for neurocysticercosis. Neurology 2001;57:177-83.

2. Rajshekhar V, Haran RP, Prakash GS, Chandy MJ. Differentiating solitary small cysticercus granulomas and tuberculomas in patients with epilepsy. Clinical and computerized tomographic criteria. J Neurosurg 1993;78:402-7.

3. Garg RK, Kar AM, Kumar T. Neurocysticercosis like presentation in a case of CNS tuberculosis. Neurol India 2000;48:260-2.

4. Garg RK. Diagnosis of intracranial tuberculoma. Indian J Tuberc 1996;43:35-9.

5. Pretell EJ, Martinot C Jr, Garcia HH, Alvarado M, Bustos JA, Martinot C; Cysticercosis Working Group in Peru. Differential diagnosis between cerebral tuberculosis and neurocysticercosis by magnetic resonance spectroscopy. J Comput Assist Tomogr 2005;29:112-4. 